\title{
Sudden death due to spontaneous right ventricular rupture: exceptional complication of arrhythmogenic right ventricular cardiomyopathy
}

\author{
Maha Sahnoun*1, Maher Jedidi ${ }^{1}$, Cherif El Khall ${ }^{1}$, Sihem Hamissa ${ }^{2}$, Moncef Mokni ${ }^{2}$, Majed \\ Zemni $^{1}$, Kamel Souguir ${ }^{1}$ \\ 1 Department of Forensic Medicine. Farhat Hached Hospital, Sousse, Tunisia \\ 2 Department of Pathology, Farhat Hached Hospital, Sousse, Tunisia \\ *Corresponding Author: Dr. Maha Sahnoun, Department of Forensic Medicine. Farhat Hached Hospital, Sousse, Tunisia. Email: \\ sahnounmaha@gmail.com; Tel: 54421086
}

Received: May 02, 2019; Accepted: June 11, 2019

\section{Abstract}

Arrhythmogenic right ventricular cardiomyopathy is characterized pathologically by a loss of myocytes and replacement by adipose and fibrous tissue. Mechanism of death mostly known is an electrical instability of right ventricular myocardium. However, a spontaneous right ventricular rupture superimposed on arrhythmogenic right ventricular cardiomyopathy is exceptional. We report a case of a 60 years old woman who died suddenly from acute heart failure complicating a spontaneous acute right ventricular rupture superimposed on arrhythmogenic right ventricular cardiomyopathy.

Keywords: Autopsy; Arrhythmogenic right ventricular dysplasia, Rupture, Spontaneous, Ventricular Dysfunction, Right.

\section{CASE REPORT}

A 60 years old woman was found dead in her bedroom. The deceased lived alone and according to her family, she had a past medical history of hypertension and a mental disorder (schizophrenia). However, the deceased did not have any history of chest pain or dyspnea.

The death was considered as suspicious and a Forensic autopsy was performed in our department of legal medicine 24 hours after death.

\section{Autopsy presentation:}

The deceased was $155 \mathrm{~cm}$ height with a well-build body. On external examination, no traumatic injuries or marks of resuscitation were noticed. Internal examination revealed a hemopericardium made by approximately $350 \mathrm{~g}$ of clotted blood (Figure 1). The heart weighted $352 \mathrm{~g}$ which demonstrated a moderate dilation of the right atrium and the right ventricle. A rupture of the myocardium which extended on $1,5 \mathrm{~cm}$ long was observed in the lateral wall of right ventricle (Figure 2) associated with a focal thinning of the right ventricular myocardium (the thickness varied between $2 \mathrm{~mm}$ and $5 \mathrm{~mm}$ ). The valvular apparatus was anatomically normal and the coronary arteries did not show any abnormalities. The right lung weighed $518 \mathrm{~g}$ and the left lung weighed $370 \mathrm{~g}$. Dissection of the lungs revealed mild edema.

No pathological findings were detected in the other organs.

Microscopic examination of the heart revealed the presence of demonstrated adipose tissue scattered diffusely throughout the right ventricular myocardium (Figure 3). No microscopic ischemic changes or inflammation were identified. Microscopic sections of the remaining internal organs did not show any abnormality. The toxicology screening was negative.

The death was attributed to acute heart failure complicating a spontaneous right ventricular rupture superimposed on Arrhythmogenic right ventricular cardiomyopathy.

\section{DISCUSSION}

Arrhythmogenic right ventricular cardiomyopathy (ARVC) is characterized pathologically by a loss of myocytes and replacement by adipose and fibrous tissue [1,2]. The clinical presentation of ARVC is highly variable, including asymptomatic 
presentations, supraventricular arrhythmias, ventricular tachycardia, right heart failure and even sudden death $[3,4]$. In the reported case, sudden death was the first manifestation of ARVC.

It has been suggested that sudden deaths due to Arrhythmogenic right ventricular cardiomyopathy occur especially in young male athletes during sports. However, several studies have reached different data. Jiao Mu et al [5] reported that sudden cardiac death caused by ARVC was contemporaneous with sports activity in only $3.5 \%$ of cases. Furthermore, both genders were equally affected. These authors demonstrated also that in spite of a peak of frequency in the 30s, Arrhythmogenic right ventricular cardiomyopathy may cause death in every period of life such as supported in this case report.

The diagnosis of ARVC remains very challenging for forensic pathologists because there are still no consensus criteria to be used to positively establish the diagnosis. Indeed, considering the presence of adipose tissue as the most important criterion is controversial. In fact, fatty infiltration is present in $50 \%$ of normal hearts in the elderly and in fatty heart [6]. While Sato T et al [7] demonstrated that the pathological pure fatty infiltration may also be a phenotype of ARVC. Even more, the fatty type was the most frequently observed, and was believed to be a higher risk for sudden death [5].

In the reported case, the only microscopic findings are an increased amount of intramyocardial adipose witch penetrates into the myocardium without demarcation. This sign argues in favor of the fatty type of ARVC [8,9]. In addition, there was no fibrosis that suggested existence of previous infraction or myocarditis and the remaining muscle cells showed no degenerative or inflammatory changes. This suggests the congenital origin of this disease [10].

All together, these data corroborate the diagnosis of arrhythmogenic right ventricular cardiomyopathy.

The most incriminated death mechanism in arrhythmogenic right ventricular cardiomyopathy remains cardiac arrhythmia. The local fatty infiltration is responsible of the retarding transmission, the development of a reentry mechanism, and the development of tachyarrhythmia leading to ventricular fibrillation and death [5]. However, heart failure complicating an acute right ventricular rupture superimposed on arrhythmogenic right ventricular cardiomyopathy is exceptional and according to our current literature search, this is the first case of rupture in this type of right ventricular cardiomyopathy. In addition, the diagnosis was established by a post-mortem examination including microscopic findings.

Right ventricular rupture occurred as a consequence of myocardial changes related to arrhythmogenic right ventricular cardiomyopathy. The association of adipose infiltration and the thickness of the right ventricular wall leaded to this complication.

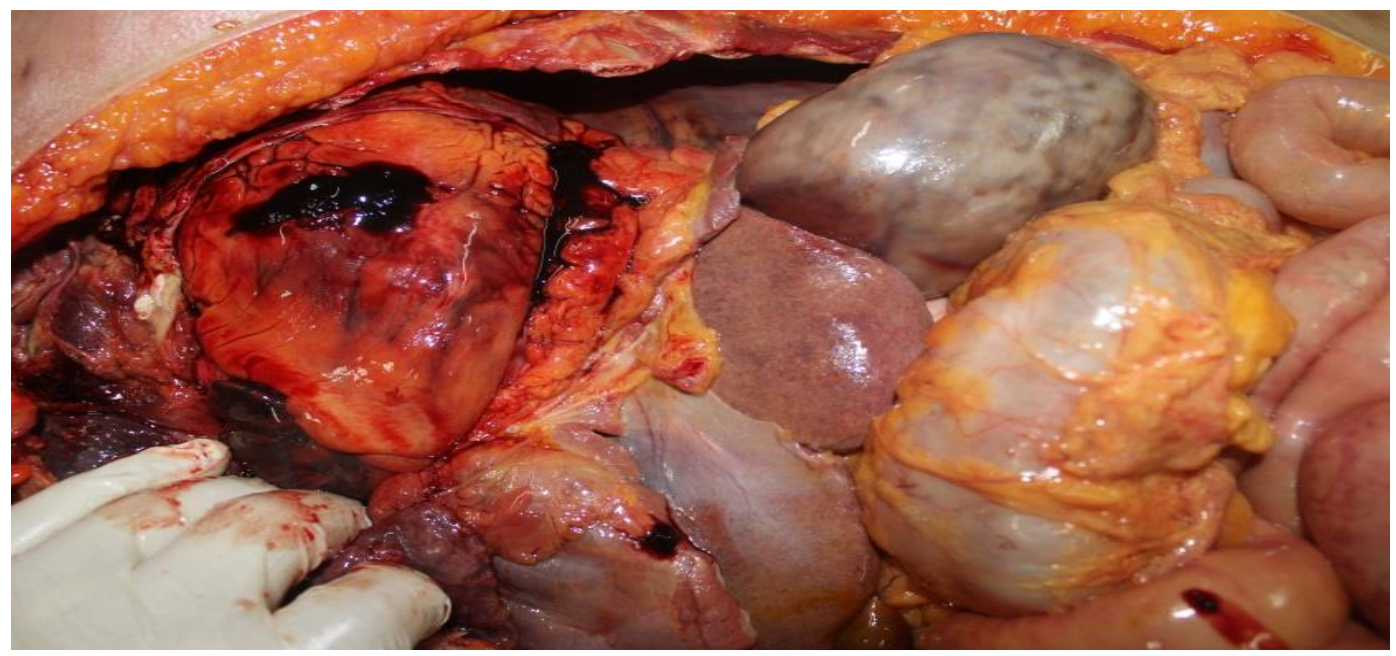

Fig 1: Hemopericardium made by coagulated blood 


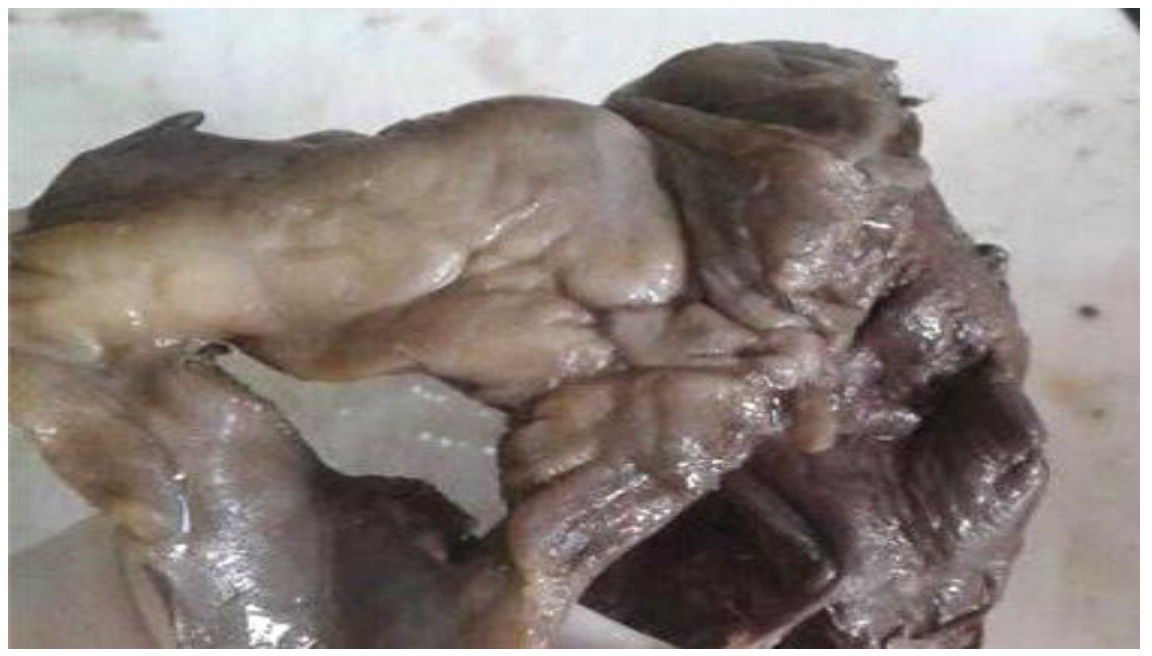

Fig 2: Gross examination of the heart: A myocardial rupture repaired in the lateral wall of right ventricle
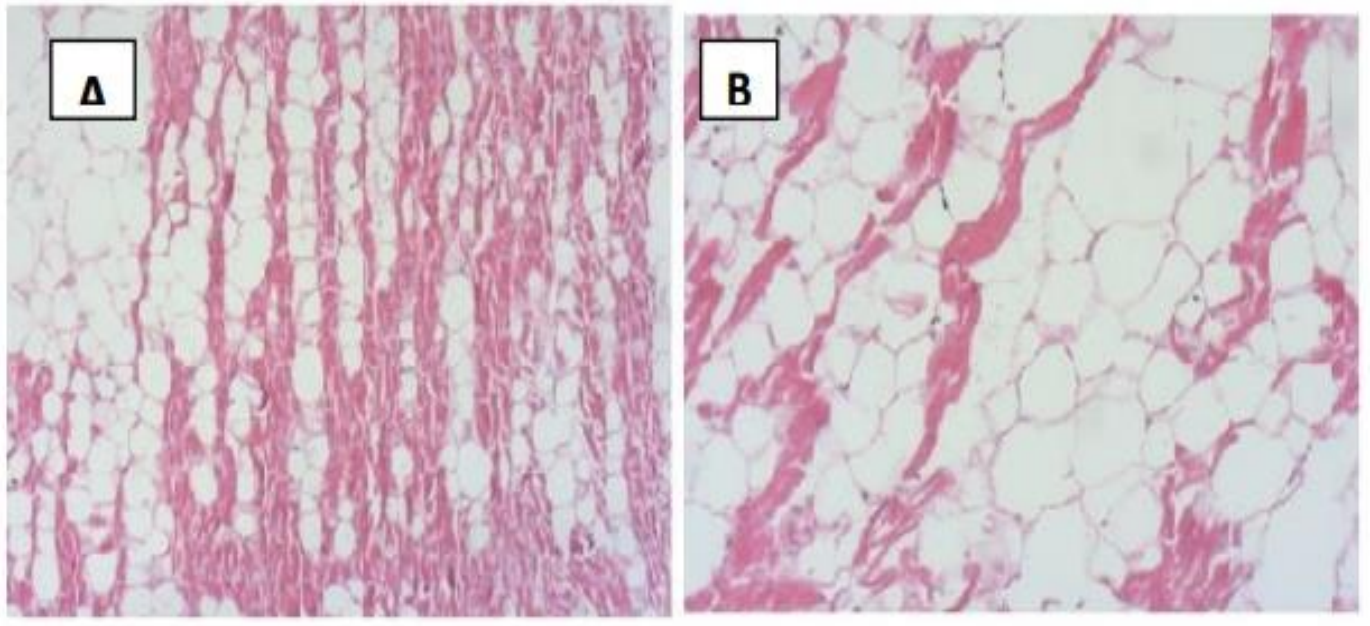

Fig 3: Photomicrography of the heart. A - Transmural fatty tissue replacement in the right free wall (hematoxylin-eosin $\times 40)$; B - High magnification view shows the residual myocardium with adipose infiltration (hematoxylin-eosin $\times 100$ )

\section{CONCLUSION}

The diagnosis of arrhythmogenic right ventricular cardiomyopathy is still challenging for forensic doctors. The most incriminated death mechanism in this cardiomyopathy is a cardiac arrhythmia. However, others mechanism should be considered as supported by the reported case.

\section{Acknowledgement}

None.

\section{Conflict of Interest}

None declared.

\section{Financial Support}

None declared.

\section{REFERENCES}

1. Fontaine G, Guiraudon G, Frank R, Vedel J, Grosgogeat Y, Cabrol C, Facquet J (1977) Stimulation studies and epicardial mapping in VT: study of mechanisms and selection for surgery. In: Kulbertus HE (ed) Reentrant arrhythmias, Chap 24. MTP Press, Lancaster, p 345.

2. Marcus F, Guiraudon G, Fontain G, et al. Right ventricular dysplasia: a report of adult cases. Circulation 1982;65:384-98.

3. Nava A, Scognamiglio R, Thiene $G$, et al. A polymorphic form of familial arrhythmogenic right ventricular dysplasia.Am J Cardiol. 1987;59: 1405-1409.

4. Fontaine G, Fontaliran F, Hebert JL. Arrhythmogenic right ventricular dysplasia. Annu Rev Med. 1999;50:17-35.

5. Jiao Mu, Guohui Zhang, DazhongXue, Mengrou Xi, Jiarui Qi,Hongmei Dong. Sudden cardiac death owing to arrhythmogenic right ventricular cardiomyopathy. Two case reports and systematic literature review. Medicine (2017) 96:47

6. Grandmaison GLDL, Bihan CL, Durigon M. Assessment of right ventricular lipomatosis by histomorphometry in control adult autopsy cases. Int J Legal Med 2001;115:105-8.

7. Sato $T$, Nishio $H$, Suzuki K. Identification of arrhythmogenic right ventricular cardiomyopathy-causing gene mutations in young 
sudden unexpected death autopsy cases. J Forensic Sci 2015;60:457-61.

8. Tabib A, Loire R, Chalabreysse L, et al. Circumstances of death and gross and microscopic observations in a series of 200 cases of sudden death associated with arrhythmogenic right ventricular cardiomyopathy and/ or dysplasia. Circulation 2003;108:3000-5.

9. Basso C, Thiene G. Adipositas cordis, fatty infiltration of the right ventricle, and arrhythmogenic right ventricular cardiomyopathy. Just a matter of fat? Cardiovascular pathology. 2005;14(1):37-41.

10. Michalodimitrakis EN, Tsiftsis DA, Tsatsakis AM, Stiakakis I. Sudden cardiac death and right ventricular dysplasia. The American journal of forensic medicine and pathology. 2001;22(1):19-22. 